\title{
Heavy quark(onium) at LHC: the statistical hadronization case
}

\author{
A Andronic ${ }^{1}, \mathbf{P}$ Braun-Munzinger ${ }^{1,2,3,4}, \mathbf{K}_{\text {Redlich }}{ }^{1,5,3}, \mathbf{J ~ S t a c h e l ~}^{6}$ \\ ${ }^{1}$ GSI Helmholtzzentrum für Schwerionenforschung, Darmstadt, Germany, \\ ${ }^{2}$ Technical University, Darmstadt, Germany ${ }^{3}$ ExtreMe Matter Institute EMMI, \\ GSI, Darmstadt, Germany ${ }^{4}$ Frankfurt Institute for Advanced Studies, J.W. Goethe \\ University, Frankfurt, Germany, ${ }^{5}$ Institute of Theoretical Physics, University of \\ Wrocław, Poland, ${ }^{6}$ Physikalisches Institut, University of Heidelberg, Germany
}

\begin{abstract}
We discuss the production of charmonium in nuclear collisions within the framework of the statistical hadronization model. We demonstrate that the model reproduces very well the availble data at RHIC. We provide predictions for the LHC energy where, dependently on the charm production cross section, a dramatically different behaviour of charmonium production as a function of centrality might be expected. We discuss also the case in elementary collisions, where clearly the statistical model does not reproduce the measurements.
\end{abstract}

\section{Introduction}

Charmonium production is considered, since the original proposal more than 20 years ago about its suppression in a Quark-Gluon Plasma (QGP) [1], an important probe to determine the degree of deconfinement reached in the fireball produced in ultrarelativistic nucleus-nucleus collisions. In the original scenario of $\mathrm{J} / \psi$ suppression via Debye screening [1] it is assumed that the charmonia are rapidly formed in initial hard collisions but are subsequently destroyed in the QGP (see recent summaries in [2, 3]).

In recent publications [4] we have demonstrated that the data on $\mathrm{J} / \psi$ and $\psi^{\prime}$ production in nucleus-nucleus collisions at the SPS $\left(\sqrt{s_{N N}} \approx 17 \mathrm{GeV}\right)$ and RHIC $\left(\sqrt{s_{N N}}=200 \mathrm{GeV}\right)$ energies can be well described within the statistical hadronization model proposed in [6] and have provided predictions for the LHC energy $\left(\sqrt{s_{N N}}=5.5\right.$ $\mathrm{TeV})$ and for energies close to threshold $\left(\sqrt{s_{N N}} \approx 6 \mathrm{GeV}\right)$. Because the mass of heavy quarks exceeds the transition temperature of the QCD phase transition by a factor of almost 8 , heavy flavor hadron production cannot be described in a purely thermal approach. It was, however, realized in [6] that charmonium and charmed hadron production can be well described by assuming that all charm quarks are produced in initial, hard collisions while charmed hadron and charmonium production takes place exclusively at the phase boundary with statistical weights calculated in a thermal approach. For a recent review of this statistical hadronization approach see [5]. An 
important element is thermal equilibration, at least near the critical temperature, $T_{c}$, which we believe can be achieved efficiently for charm only in the QGP.

An important question is whether the statistical behavior is a unique feature of high energy nucleus-nucleus collisions or whether it is also encountered in elementary collisions, where finite size effects will obscure a possible phase transition. In early analyses (see ref. [7]), it is indeed argued that hadron production in $\mathrm{e}^{+} \mathrm{e}^{-}$and pp is thermal in nature. Furthermore, such analyses of hadron multiplicities (for recent results see [8, 9, 10] and refs. therein) yield also temperature values in the range of 160-170 $\mathrm{MeV}$.

To establish the uniqueness of the $\mathrm{J} / \psi$ probe for the diagnosis of a QGP in nucleusnucleus collisions it is important to understand whether similar thermal features as observed in nucleus-nucleus collisions are also at work in heavy-flavor hadron production in elementary collisions. We have recently demonstrated [11] that there are crucial differences in elementary collisions compared to nucleus-nucleus.

\section{The statistical model in elementary collisions}

For the study of hadron production in $\mathrm{e}^{+} \mathrm{e}^{-}$collisions we employ the canonical statistical model described in [8, 11] (see also [9, 12]). For the present study, we perform calculations for two cases: i) a 2-jet initial state which carries the quantum numbers of the 5 flavors, with the relative abundance of the five flavors in one jet and corresponding antiflavor in the other jet taken form the measurements at the $Z^{0}$ resonance quoted in [13. These relative abundances $(17.6 \%$ for $u \bar{u}$ and $c \bar{c}$ and $21.6 \%$ for $d \bar{d}, s \bar{s}$ and $b \bar{b}$ ) are thus external input values, unrelated with the thermal model. ii) a purely thermal ansatz, i.e. a 2-jet initial state characterized by vanishing quantum numbers in each jet.

In Fig. 1] we show a comparison of data [13] and model prediction for charmed and bottom hadron yields in $\mathrm{e}^{+} \mathrm{e}^{-}$annihilations at $\sqrt{s}=91 \mathrm{GeV}$. For the model we have used the parameter set: $T=170 \mathrm{MeV}, V=16 \mathrm{fm}^{3}$ and $\gamma_{s}=0.66$, which represents the best fit of multiplicities of hadrons with lighter quarks [8].

We first note that the calculation employing the 5-flavor scheme is in very good agreement with the data, as demonstrated by the good $\chi^{2}$ per degree of freedom between the model and the data (excluding the $\Upsilon$ and prompt $\mathrm{J} / \psi$ ) of $21.7 / 16(34 / 18$ when including all species) (see also ref. [9]). Despite this overall agreement, the exceptions are significant: the $\Upsilon$ meson yield is underpredicted by the model by 17 orders of magnitude, while the prompt $\mathrm{J} / \psi$ yield [14] is underpredicted by almost 2 orders of magnitude. Obviously, the production of quarkonia is expected to be strongly suppressed in the statistical model. The disagreement is a consequence of the separate hadronization of the $c$ and $\bar{c}$ quarks. The measured prompt $\mathrm{J} / \psi$ production in $Z^{0}$ decays (into hadrons) is about $3 \times 10^{-4}\left[14\right.$. The thermal model predicts a prompt yield for $\mathrm{J} / \psi$ of $4.1 \times 10^{-6}$ $\left(1.6 \times 10^{-7}\right.$ for $\psi^{\prime}$ and $4.3 \times 10^{-7}$ for $\left.\chi_{c 1}\right)$, identically for the two calculation schemes. Whenever the model seems to describe the yields of charmonia the measured yields are 


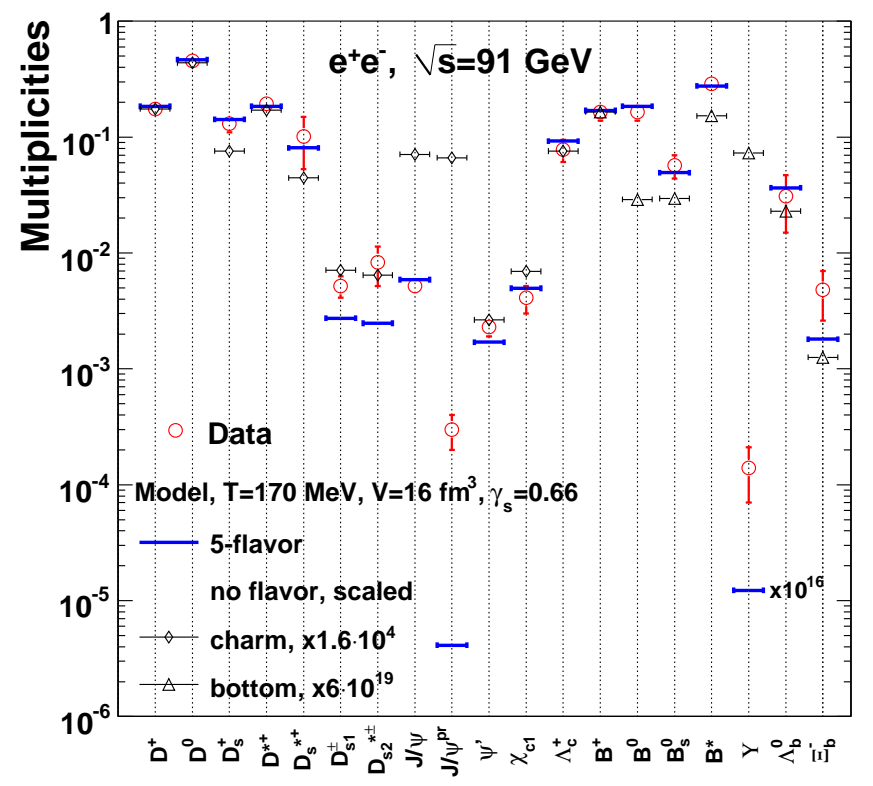

Figure 1. Multiplicities of hadrons with charm and bottom quarks in $\mathrm{e}^{+} \mathrm{e}^{-}$collisions compared to the thermal model calculations for two cases: i) the 5flavor jet scheme (thick lines) and ii) no (net) flavor jet scheme (thin lines with diamonds for the charm sector and triangles for bottom). Note, for case ii) the large factors used to scale the model calculations to fit in the plotting range. The data are from the compilation published by the Particle Data Group (PDG) 13. The prompt $\mathrm{J} / \psi$ measurement $\mathrm{J} / \psi^{p r}$ is from the L3 experiment [14].

dominated by the feed down from bottom hadrons and the agreement only reflects the agreement seen for the open bottom hadrons and their branching ratios to charmonia, properly considered in the model.

The calculation employing a purely thermal ansatz underpredicts all the measurements by many oders of magnitude, while for the light quark sector the differences between calculations with a pure thermal model and with the 5-flavor quarkantiquark scheme were found to be small [8]. The strangeness suppression factor, which for the present results only enters in the calculation of the yields of $D_{s}$ and $B_{s}$ mesons, appears to have no counterpart in the heavy quark sector. This reflects the fact that a negligible number of $c$ and $b$ quarks are formed in the fragmentation process. In this case, the thermal weights describe the distribution of the initial quarks into heavy flavor hadrons. Chemical equilibration is not required in this process.

For the case of hadron production in elementary hadronic collisions we employ the canonical realization of the thermal model [15, 4, 16]. For the description of the relative production cross sections of heavy flavored hadrons, the energy dependence of the temperature parameter is the only model input, which is taken in a parametrized form from the fits of $(u, d, s)$-hadron abundancies in central nucleus-nucleus collisions [17]. For c.m. energies beyond $10 \mathrm{GeV}$ per nucleon pair in nucleus-nucleus collisions a limiting temperature $T_{\text {lim }}=164 \pm 5 \mathrm{MeV}$ is reached. Recent fits of hadron yields in pp collisions [10] give very similar values, independent of anergy.

In Fig. 2 we show the model comparison to data for the relative production cross section of $\psi^{\prime}$ and $J / \psi$ charmonia $\$$. The measurements in $\mathrm{pA}$ and $\mathrm{pp}(\overline{\mathrm{p}})$ collisions are above the model values by about a factor 4 (corresponding to 10 experimental standard

$\ddagger$ The charm production cross section, which is an important model input parameter for the calculations of absolute yields, cancels out for this ratio. 


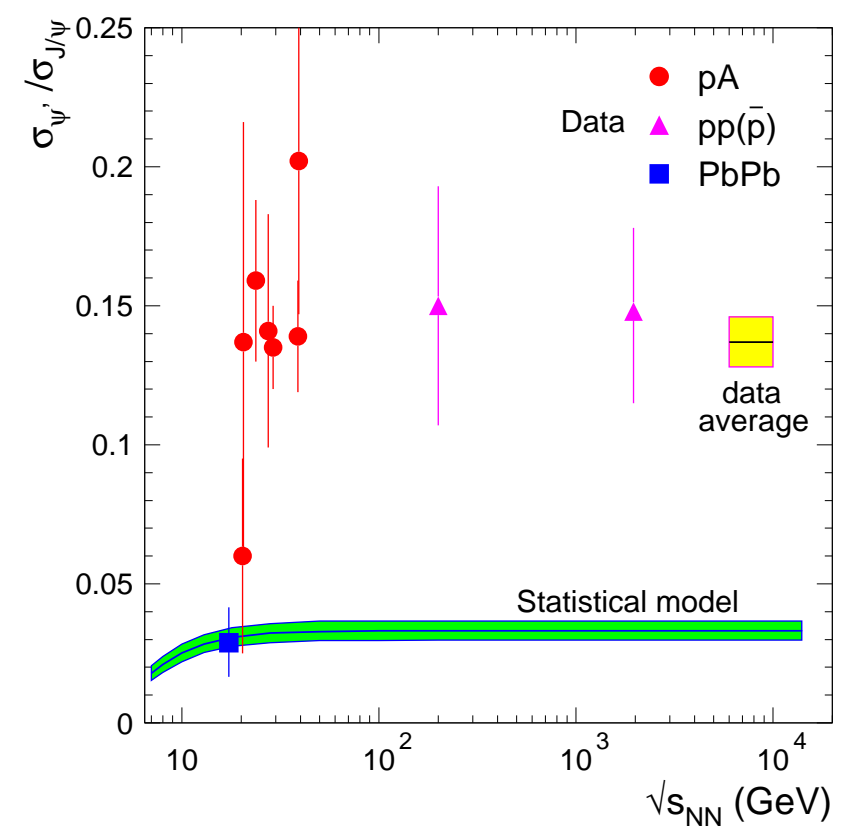

Figure 2. Production cross section of $\psi^{\prime}$ relative to $J / \psi$. The data for pA collisions are from the compilation by Maltoni et al. [19]; the points for elementary collisions are from the PHENIX experiment at RHIC [20] and from the CDF experiment at Tevatron 22] (see text); the data point for $\mathrm{Pb}+\mathrm{Pb}$ collisions at the SPS energy is from the NA50 experiment [18. The average value of the $\mathrm{pA}$ and $\mathrm{pp}(\overline{\mathrm{p}})$ measurements with the corresponding error (see text) is represented by the shaded box. The band denotes statistical model calculations for the temperature parametrization from heavy-ion fits [17] $\left(T_{l i m}=164 \mathrm{MeV}\right)$ with $\pm 5 \mathrm{MeV}$ error.

deviations; the average value of the measurements is $0.137 \pm 0.009$, with a $\chi^{2}$ per degree of freedom of 0.88 ). The relative production cross sections of charmonium states, as are observed in all measurements in hadronic collisions cannot be described in the thermal approach. The temperature needed to explain the data would be $300 \mathrm{MeV}$, well above the Hagedorn limiting temperature, which is about $200 \mathrm{MeV}$. This is in sharp contrast to the (only currently existing) measurement in central nucleus-nucleus collisions, performed at the SPS by the NA50 experiment [18, which is well described. We note that the $\mathrm{pA}$ data exhibit a constant $\psi^{\prime} / J / \psi$ production ratio as a function of energy. In the model, the value is determined only by the temperature and this is reflected in the slight decrease of the ratio towards low energies. A constant value, also up to the LHC energies, is predicted beyond $\sqrt{s_{N N}} \simeq 20 \mathrm{GeV}$.

The measurements reported in Fig. 2 demonstrate that the relative production cross section $\psi^{\prime} / J / \psi$ is identical in $\mathrm{pA}$ and in $\mathrm{pp}(\overline{\mathrm{p}})$ collisions, implying no visible influence of the cold nuclear medium. Note that the ratio for the Tevatron energy was derived from the CDF measurements of $J / \psi$ [21] and $\psi^{\prime}$ [22] and is for transverse momentum

$p_{t}>1.25 \mathrm{GeV} / \mathrm{c}$ (we have extrapolated the $\psi^{\prime}$ measurement from $2 \mathrm{GeV} / \mathrm{c}$ down to 1.25 $\mathrm{GeV} / \mathrm{c})$.

\section{The statistical hadronization model in nucleus-nucleus collisions}

The model has the following input parameters: i) characteristics at chemical freeze-out: temperature, $T$, baryochemical potential, $\mu_{b}$, and volume corresponding to one unit of rapidity $V_{\Delta y=1}$, extracted from thermal fits of non-charmed hadrons [17]; ii) the charm production cross section in pp collisions, taken either from NLO pQCD calculations [23, 24] or from experiment [25], used to calculate the number of directly produced $c \bar{c}$ pairs $N_{c \bar{c}}^{d i r}$. The heavy quark (charm or bottom) balance equation [6] (including 
canonical suppression factors [15, 4], $\left.I_{1} / I_{0}\right)$ :

$$
N_{c \bar{c}}^{d i r}=\frac{1}{2} g_{c} N_{o c}^{t h} \frac{I_{1}\left(g_{c} N_{o c}^{t h}\right)}{I_{0}\left(g_{c} N_{o c}^{t h}\right)}+g_{c}^{2} N_{c \bar{c}}^{t h} .
$$

is used to determine the fugacity factor $g_{c}$ ( $I_{n}$ are modified Bessel functions; $N_{o c}^{t h}$ and $N_{c \bar{c}}^{t h}$ are the numbers of open and hidden charm hadrons, respectively, in the volume $V_{\Delta y=1}$, computed from their grand-canonical densities).

A comprehensive set of model predictions as a function of energy, from the charm production threshold up to the LHC, is presented in Fig. 3 for central Au-Au collisions $\left(N_{\text {part }}=350\right)$. The left panel shows our predictions for the energy dependence of midrapidity yields for various open and hidden charm hadrons. The most striking behavior is observed for the production of $\Lambda_{c}^{+}$baryons: their yield relative to other charmed hadrons rises significantly towards lower energies. In our approach this is caused by the increase in baryochemical potential towards lower energies (coupled with the charm neutrality condition). A similar behavior is seen for the $\Xi_{c}^{+}$baryon. The relative production yields of D-mesons depend on their quark content and depend on energy only around threshold. These results emphasize the importance of measuring, at low energies, in addition to D-mesons, also the yield of charmed baryons to get a complete measure of the total charm production cross section.
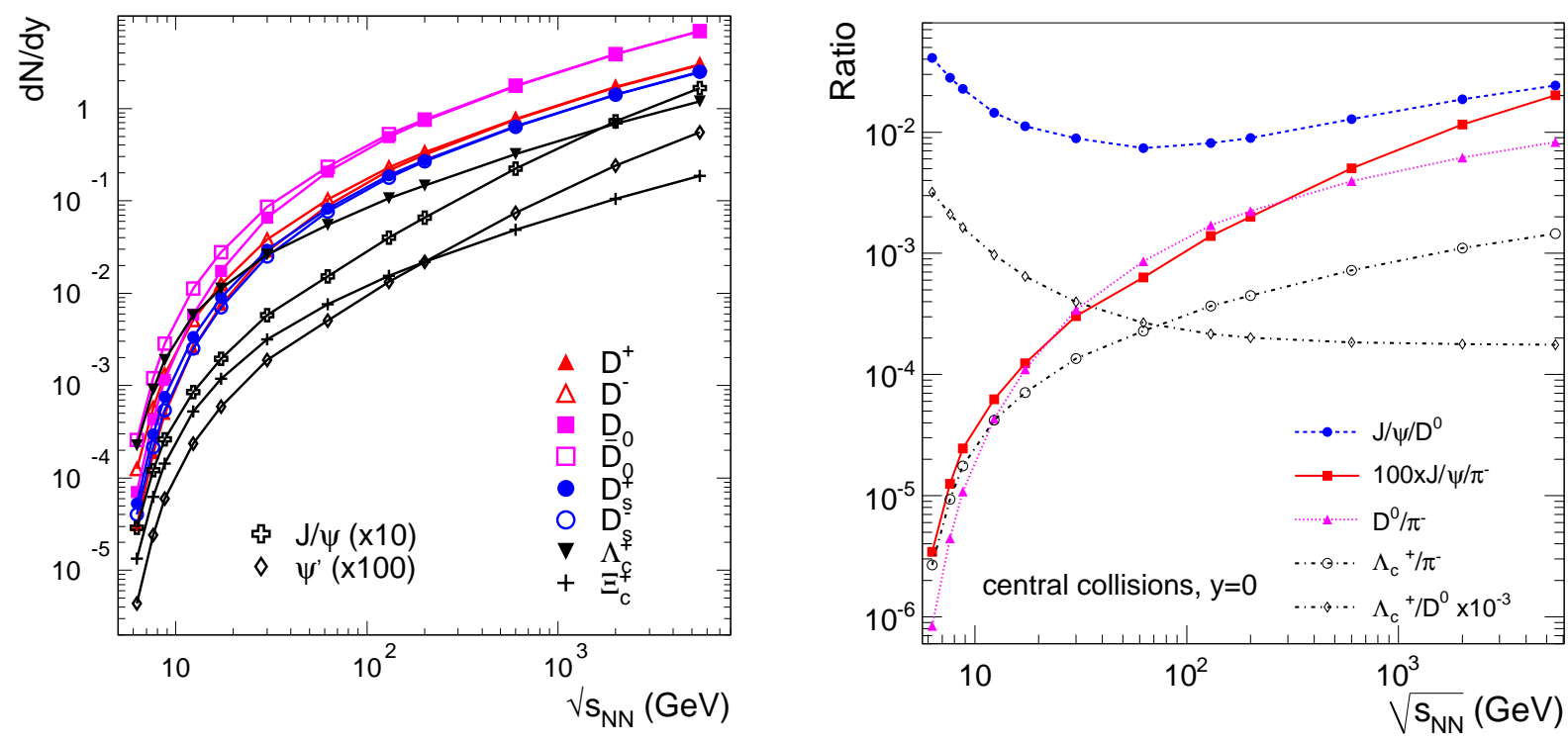

Figure 3. Left panel: energy dependence of the production yield at midrapidity for open and hidden charm hadrons (with the energy dependence of $\mathrm{d} \sigma_{c \bar{c}} / \mathrm{d} y$ as in [4]). Right panel: ratios calculated from midrapidity yields.

One of the motivations for the study of charm production at low energies was the expectation [26, 27] to provide, by a measurement of D-meson production near threshold, information on their possible in-medium modification near the phase boundary. However, the cross section $\sigma_{c \bar{c}}$ is governed by the mass of the charm quark $m_{c} \approx 1.3 \mathrm{GeV}$, which is much larger than any soft QCD scale such as $\Lambda_{Q C D}$. 
Therefore we expect no medium effects on this quantity. The much later formed Dmesons, or other charmed hadrons, may well change their mass in the hot medium. Whatever the medium effects may be, they can, because of the charm conservation, $\sigma_{c \bar{c}}=\frac{1}{2}\left(\sigma_{D}+\sigma_{\Lambda_{c}}+\ldots\right)+\left(\sigma_{\eta_{c}}+\sigma_{J / \psi}+\ldots\right)$, expressed in Eq. (11), in first order only lead to a redistribution of charm quarks [4. In contrast, as a consequence of in-medium masses of open charm hadrons, the yields of charmonia can vary by up to $40-50 \%$ and this is more prominent at threshold energies [4].

In the right panel of Fig. 3 we show the energy dependence of ratios of mid-rapidity yields of open and hidden charm hadrons and pions. The relative production of charmed hadrons to pions exhibits a monotonic dependence on energy, unlike equivalent ratios involving strange hadrons [17]. The different bahavior compared to the strangeness sector is mainly due to the strong canonical suppression of open charm at low energies (determined in turn by strong decrease of the charm production cross section at low energies). A non-monotonic behavior is observed in the production ratio of $J / \psi$ to $D^{0}$, determined by the interplay of the strong canonical suppression of open charm at low energies and the gradual onset as a function of energy of the quadratic term in Eq. (1).

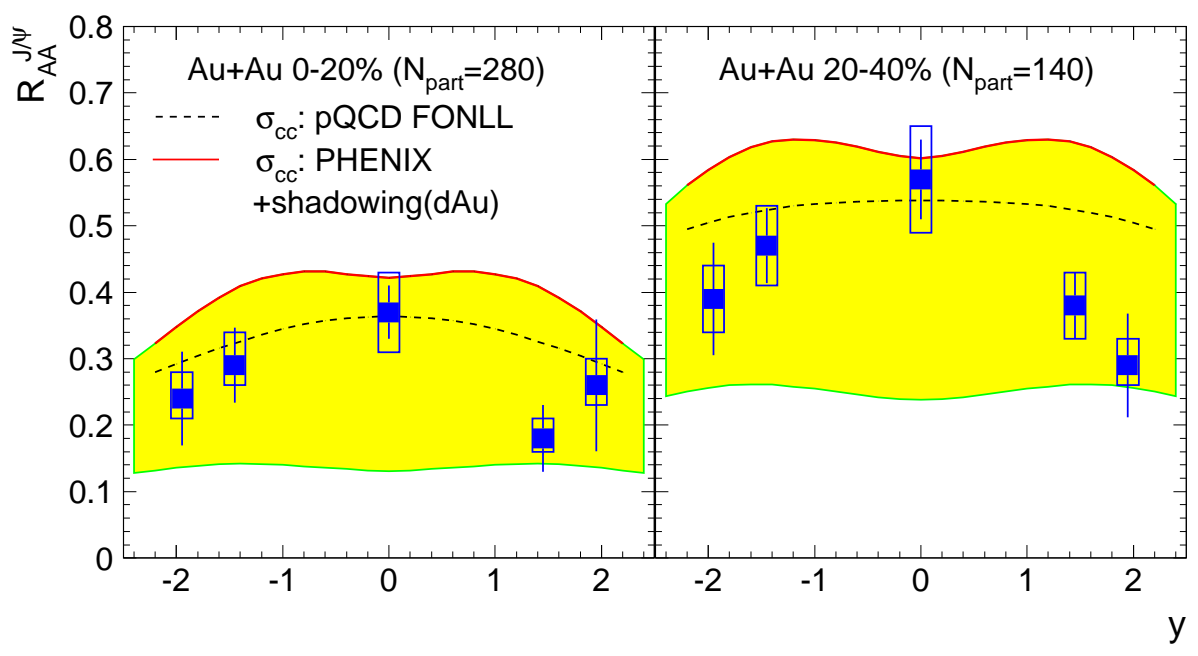

Figure 4. Rapidity dependence of $R_{A A}^{J / \psi}$ for two centrality classes. The data from the PHENIX experiment 28] (symbols with errors) are compared to calculations (lines, see text). The shaded area corresponds to calculations for the lower limit of the charm cross section as measured by PHENIX [25, with our shadowing scenario.

In Fig. 4 we present the rapidity dependence of the nuclear modification factor $R_{A A}^{J / \psi}$ (we use experimental data for the pp reference). While earlier [4] we have compared data [28] to our model predictions for the pQCD charm production cross section [23] (dashed line in Fig. (4), we use here as alternative the charm cross section as measured by PHENIX in pp collisions [25] and consider in addition shadowing for $\mathrm{Au}-\mathrm{Au}$ collsions as extracted from recent dAu data [29]; we assume that the deviation of $R_{d A u}^{J / \psi}$ from unity is entirely due to shadowing. Also in this case, our model describes the observed suppression and its rapidity dependence. The maximum of $R_{A A}^{J / \psi}$ at midrapidity is in our model due to the enhanced generation of charmonium around mid-rapidity, determined 
by the rapidity dependence of the charm production cross section. In this sense, the above result constitutes strong evidence for the statistical generation of $\mathrm{J} / \psi$ at chemical freeze-out.
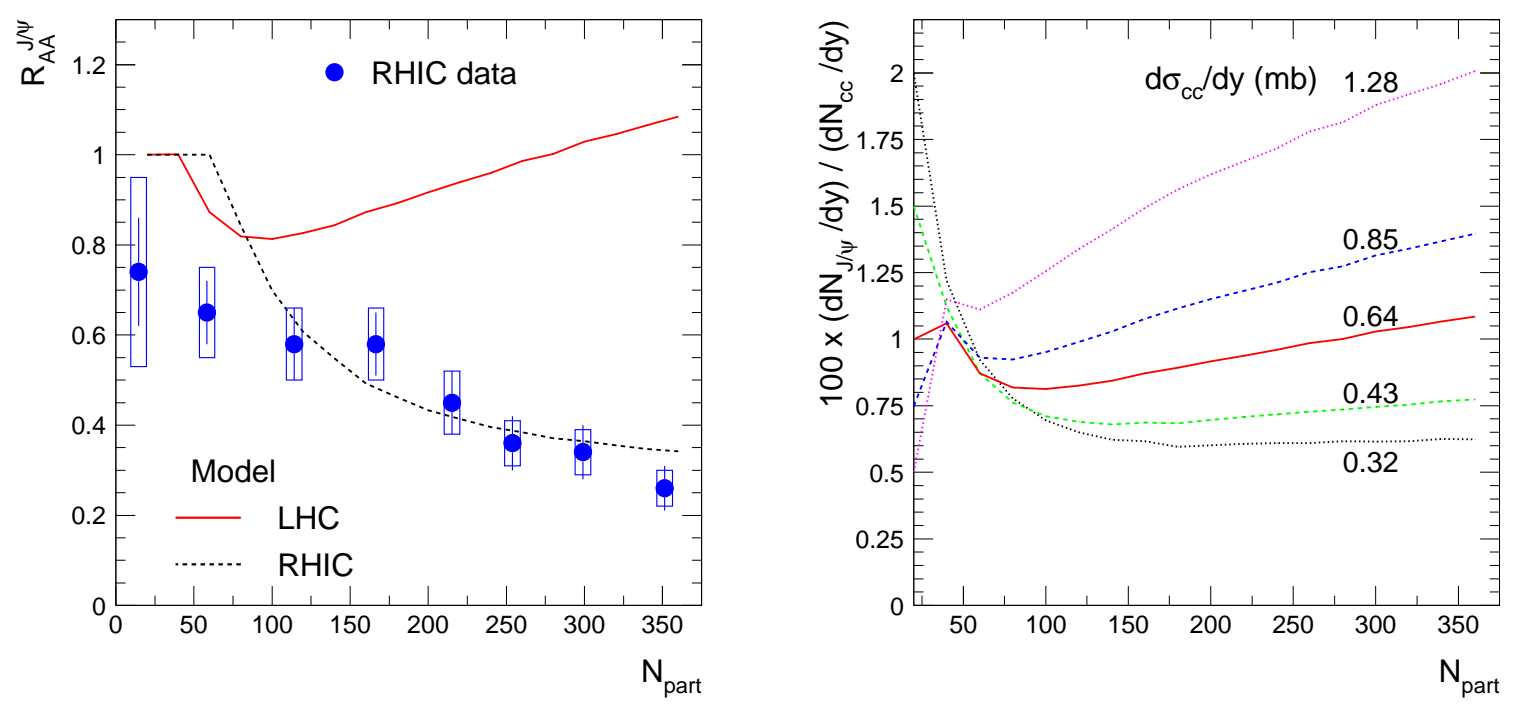

Figure 5. Centrality dependence of $R_{A A}^{J / \psi}$ for RHIC and LHC energies (left panel) and of the $J / \psi$ rapidity density at LHC relative to the number of initially produced $c \bar{c}$ pairs (right panel, curves labelled by the $\mathrm{d} \sigma_{c \bar{c}} / \mathrm{d} y$ ) at midrapidity.

The centrality dependence of $R_{A A}^{J / \psi}$ at midrapidity is shown in the left panel of Fig. 5. Our calculations approach the value in pp collisions around $N_{\text {part }}=50$, which corresponds to an assumed minimal volume for the creation of QGP of $400 \mathrm{fm}^{3}$ [4]. The model reproduces very well the decreasing trend versus centrality seen in the RHIC data [28]. Note that, in our model, the centrality dependence of the nuclear modification factor arises entirely as a consequence of the still rather moderate rapidity density of initially produced charm quark pairs at RHIC $\left(\mathrm{d} N_{c \bar{c}} / \mathrm{d} y=1.6\right.$ for central collisions, using the FONLL charm production cross section [23]) leading to a marked centrality dependence of the canonical suppression factor for open charm hadrons. At the much higher LHC energy the charm production cross section (including shadowing in $\mathrm{PbPb}$ collisions 24]) is expected to be about an order of magnitude larger. As a result, the opposite trend as a function of centrality is predicted, with $R_{A A}^{J / \psi}$ exceeding unity for central collisions. A significantly larger enhancement of about a factor of 2 is obtained if the charm production cross section is two times larger than presently assumed, as seen in the right panel of Fig. [5, where we show the $J / \psi$ production relative to the number of initially produced $c \bar{c}$ pairs.

\section{Conclusions}

We have shown that the statistical hadronization model describes well the measured decrease with centrality and the rapidity dependence of $R_{A A}^{J / \psi}$ at RHIC energy. 
Importantly, well described in central heavy-ion collisions at SPS energy, where we have the only measurement to date, is the ratio $\psi^{\prime} / J / \psi$. In contrast, in elementary, $\mathrm{e}^{+} \mathrm{e}^{-}$and in pp and $\mathrm{p}(\pi)$-nucleus, collisions the charmonium measurements cannot be explained within the statistical model. This underlines the relevance of the statistical features in nucleus-nucleus collisions. Extrapolation to LHC energy leads, contrary to the observations at RHIC, to $R_{A A}^{J / \psi}$ increasing with collision centrality. If observed, such a behavior will provide a dramatic proof of charmonium as the ultimate observable to delineate the phase boundary of QCD matter.

K.R. acknowledges support from the Alexander von Humboldt Foundation.

\section{References}

[1] T. Matsui, H. Satz, Phys. Lett. B 178 (1986) 416.

[2] L. Kluberg, H. Satz, arXiv:0901.3831 [hep-ph].

[3] R. Rapp, D. Blaschke, P. Crochet, arXiv:0807.2470 [hep-ph].

[4] A. Andronic, P. Braun-Munzinger, K. Redlich, J. Stachel, Nucl. Phys. A 789 (2007) 334; Phys. Lett. B 652 (2007) 259; Phys. Lett. B 659 (2008) 149.

[5] P. Braun-Munzinger, J. Stachel, arXiv:0901.2500,

[6] P. Braun-Munzinger, J. Stachel, Phys. Lett. B 490 (2000) 196; Nucl. Phys. A 690 (2001) 119c.

[7] F. Becattini, arXiv:hep-ph/9701275 J. Phys. G 23 (1997) 1933.

[8] A. Andronic, F. Beutler, P. Braun-Munzinger, K. Redlich, J. Stachel, Phys. Lett. B 675 (2009) 312.

[9] F. Becattini, P. Castorina, J. Manninen, H. Satz, Eur. Phys. J. C 56 (2008) 493.

[10] I. Kraus, J. Cleymans, H. Oeschler, K. Redlich, Phys. Rev. C 79 (2009) 014901.

[11] A. Andronic, F. Beutler, P. Braun-Munzinger, K. Redlich, J. Stachel, Phys. Lett. B 678 (2009) 350.

[12] F. Beutler, A. Andronic, P. Braun-Munzinger, K. Redlich, J. Stachel, arXiv:0910.1697.

[13] C. Amsler et al. (Particle Data Group), Phys. Lett. B 667 (2008) 1.

[14] M. Acciarri et al. (L3 coll.), Phys. Lett. B 453 (1999) 94.

[15] M.I. Gorenstein, A.P. Kostyuk, H. Stöcker, W. Greiner, Phys. Lett. B 509 (2001) 277.

[16] L. Grandchamp, R. Rapp, Phys. Lett. B 523 (2001) 60; Nucl. Phys. A 709 (2002) 415.

[17] A. Andronic, P. Braun-Munzinger, J. Stachel, Phys. Lett. B 673 (2009) 142; Erratum: ibid. 673 (2009) 516.

[18] B. Alessandro et al. (NA50 coll.), Eur. Phys. J. C 49 (2007) 559.

[19] F. Maltoni et al., Phys. Lett. B 638 (2006) 202.

[20] PHENIX collaboration, results presented at Quark Matter 2009 conference, http://www.phy.ornl.gov/QM09/

[21] D. Acosta et al. (CDF coll.), Phys. Rev. D 71 (2005) 032001.

[22] T. Aaltonen et al. (CDF coll.), Phys. Rev. D 80 (2009) 031103.

[23] M. Cacciari, P. Nason, R. Vogt, Phys. Rev. Lett. 95 (2005) 122001.

[24] ALICE Collaboration, J. Phys. G 32 (2006) 1295; R. Vogt, Int. J. Mod. Phys. E 12 (2003) 211.

[25] A. Adare et al. (PHENIX coll.), Phys. Rev. Lett. 97 (2006) 252002.

[26] P. Senger, J. Phys. Conf. Series 50 (2006) 357.

[27] L. Tolos, J. Schaffner-Bielich, H. Stöcker, Phys. Lett. B 635 (2006) 85.

[28] A. Adare et al. (PHENIX coll.), Phys. Rev. Lett. 98 (2007) 232301.

[29] A. Adare et al. (PHENIX coll.), Phys. Rev. C 77 (2008) 024912; Erratum ibid. C 79 (2009) 059901 